\title{
How the matrix metalloproteinase MMP14 contributes to the progression of colorectal cancer
}

\author{
Lena Claesson-Welsh \\ Uppsala University, Beijer and Science for Life Laboratories, Department of Immunology, Genetics and Pathology, Uppsala, Sweden.
}

\begin{abstract}
Certain matrix metalloproteinase (MMP) family proteins have been associated with cell proliferation and invasion in aggressive cancers. However, attempts to target the MMPs with the hope of treating tumors have thus far failed. In this issue of the $\mathrm{JCl}$, Ragusa and coworkers identified an intestinal cancer subgroup of slow-growing, chemotherapy-resistant, and very aggressive matrix-rich tumors that mimic a hard-to-treat colorectal cancer subtype in humans. These tumors showed downregulated levels of the transcription factor prospero homeobox protein 1 (PROX1), which relieved repression of the matrix metalloproteinase MMP14. Upregulated MMP14 levels correlated with blood vessel dysfunction and a lack of cytotoxic T cells. Notably, blockade of proangiogenic factors in combination with stimulation of the CD40 pathway in the mouse cancer model boosted cytotoxic T cell infiltration. The study illustrates how combinatorial treatments for aggressive, $\mathrm{T}$ cell-deficient cancers can launch an antitumor immune response.
\end{abstract}

\section{The matrix metalloproteinase} family

The discovery of an amphibian interstitial collagenase by Gross and Lapiere in 1962 sparked intense research into the structure and biology of a 25-member family of clinically relevant, calciumdependent, zinc-containing endopeptidases in humans: the matrix metalloproteinase (MMP) family $(1,2)$. The welldescribed matrix-degrading activities of MMPs result in the release of a wide range of bioactive molecules. These may be generated from the matrix itself but also include various growth-regulatory molecules, such as growth factors, that are stored in the matrix. In addition, substrates for MMP protease activity include a range of non-matrix molecules (3). Certain MMPs such as the transmembrane MMP14 (also termed MT1-MMP) have been strongly linked to cell proliferation and invasion (4-6). However, clinical cancer trials of MMP-targeting drugs have thus far been unsuccessful (7).

Now, the elegant study by Ragusa and coworkers (8) describes how the intricate activities of MMP14, unleashed by loss of the transcription factor prospero homeobox protein 1 (PROX1), contributes to the progression of colorectal cancer (CRC). Intratumoral fluctuations in signaling through tumoral WNT and Notch pathways trigger a decrease in PROX1 levels and lead to MMP14 upregulation (Figure 1), propelling a series of unfavorable changes in the tumor microenvironment. PROX1 is a transcriptional repressor of MMP14, which, notably, is the only known target for the repressive effects of PROX1 (9). The clinical relevance of these findings is exemplified by the improved survival of patients with microsatellite-stable CRC exhibiting high PROX1 and low MMP14

Related Article: p. 1199

Conflict of interest: The author has declared that no conflict of interest exists.

Copyright: (c) 2020, American Society for Clinical Investigation.

Reference information: / Clin Invest. 2020;130(3):1093-1095. https://doi.org/10.1172/JCI135239.

expression (8). What happens in the lowPROX1-expressing tumors? This is now resolved by Ragusa and coworkers, who used a range of genetic mouse models in which intestinal cancer developed spontaneously upon deletion of the CRC hallmark genes adenomatous polyposis coli (APC) and p53. When the researchers went on to delete Prox1, MMP14 was induced, and the mice developed slowgrowing, matrix-rich, chemotherapy-resistant tumors with a sinister stromal signature: fibroblasts became activated, blood vessels lost their function, and cytotoxic $\mathrm{T}$ cells failed to enter the tumor.

\section{Luring T cells to the tumor}

The rapid clinical implementation of cancer immune therapy, the development for which James Allison and Tasuko Honjo were awarded the Nobel Prize in 2018, has put the spotlight on cytotoxic T cells (CD8 ${ }^{+}$ $\mathrm{T}$ cells). The so-called checkpoint inhibitors, developed by Allison and Honjo, prompt $\mathrm{CD}^{+} \mathrm{T}$ cell killing of tumor cells. In several human cancer types, in particular melanoma, the use of checkpoint inhibitors, such as Abs against the checkpoint protein programmed cell death (PD) ligand or receptor, has had remarkable effects in promoting long-term survival and perhaps even cure (10). In contrast, only a small fraction of patients with CRC benefit from treatment with checkpoint inhibitors. The simple explanation for the treatment failure is that the immune therapy-resistant CRC subtype has no or too few $\mathrm{CD}^{+} \mathrm{T}$ cells in the tumor (11). It's a straightforward conclusion: cancers that allow $\mathrm{CD}^{+} \mathrm{T}$ cell infiltration can respond to checkpoint inhibitors, whereas those without $\mathrm{CD}^{+} \mathrm{T}$ cells fail to respond. The urgent task, therefore, is to find out how to lure $\mathrm{T}$ cells to the tumor and facilitate their way across the wall of tumor blood vessels and into the tissue to do their job: killing off tumor cells.

What stops $\mathrm{T}$ cells from infiltrating the low-PROX1-/high-MMP14-expressing 


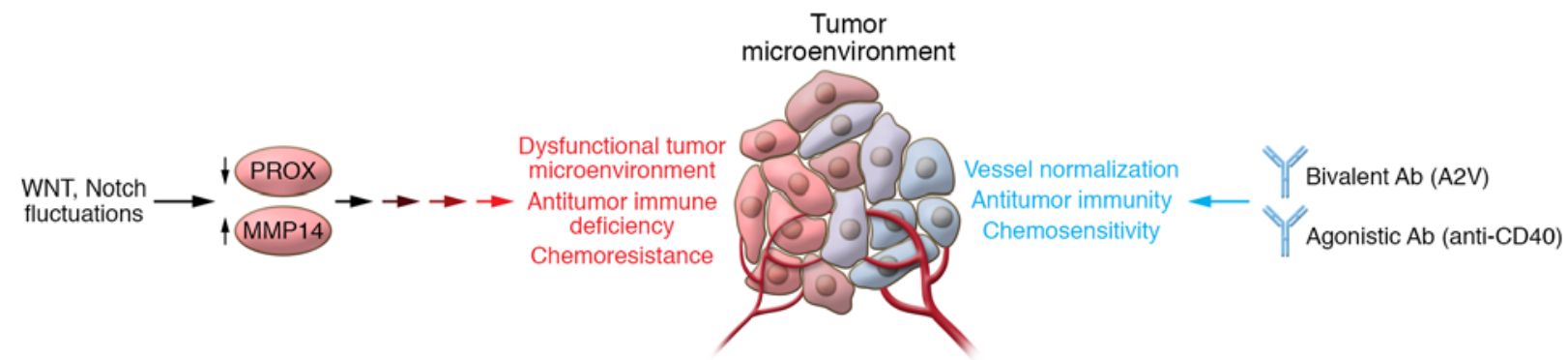

Figure 1. PROX1/MMP14 in intestinal cancer. Intratumoral fluctuations in signaling through tumoral WNT and Notch pathways trigger a decrease in PROX1 levels. Loss of PROX1 releases MMP14, which propels the development of an unfavorable tumor microenvironment, loss of antitumor immunity, and chemoresistance. Normalization of tumor blood vessels with bivalent anti-VEGFA/anti-ANGPT2 (A2V) Abs combined with agonistic anti-CD40 Ab promotes antitumor immunity and a gain of chemosensitivity.

tumors? One obvious culprit would be the MMP14-regulated matrix. The matrix regulates tissue stiffness and is likely to directly contribute to the dysfunctionality of cancer-associated fibroblasts and tumor blood vessels and to the lack of antitumor immune cells by inadvertent release of growth-regulatory factors and cytokines. The well-documented dysfunctionality of tumor blood vessels due to excessive stimulation by vascular endothelial growth factor (VEGFA) and angiopoietin 2 (ANGPT2) causes vessel barrier breakdown and leakage of blood constituents into the tumor tissue. One would assume that barrier deterioration would facilitate immune cell infiltration, but this does not seem to be the case. Instead, for $\mathrm{T}$ cells to make their way into the tumor, they need to attach to a well-organized vessel surface. The concept of vessel "normalization," achieved, for example, by neutralization of VEGFA using anti-VEGFA Abs, has been introduced to describe the morphology of a less stimulated and more functional tumor vasculature (12).

Importantly, to normalize the vasculature, the authors used a bivalent $\mathrm{Ab}$, A2V (13), that recognizes both VEGFA and ANGPT2. Whereas treatment with anti-VEGFA Abs (bevacizumab) provides well-documented but limited benefits for patients with metastatic CRC (14), antiANGPT2 therapy has thus far not progressed in trials, although many are still ongoing (15). The potential clinical gains of $\mathrm{A} 2 \mathrm{~V}$, or other means to simultaneously block VEGFA and ANGPT2, are currently being tested. Interestingly, preclinical data indicate that the outcome will be dependent on the tumor microenvironment, such as the nature of the lymphoid or myeloid cell populations (15). Indeed, in the intestinal cancer models tested in the Ragusa study (8), combined treatment with the neutralizing A2V Abs and agonistic CD40 Abs resulted in vessel normalization, increased $\mathrm{CD}^{+} \mathrm{T}$ cell infiltration, and tumor cell death. CD40 Abs may have broad effects, as CD40 is known to be expressed on tumor-associated macrophages, DCs, and B cells. Through DCs, the agonistic CD40 Abs may indirectly boost $\mathrm{CD}^{+} \mathrm{T}$ cell activity (16). Ragusa and coworkers also found substantial CD40 expression on cancer fibroblasts, implying a broad range of effects through this pathway (8). A2V/CD40 Ab-treated tumors, moreover, contained high-endothelial venule-like (HEV-like) clusters with B and $\mathrm{T}$ cells, which have been associated with antitumor immunity and a less aggressive disease (17).

\section{Clinical implications}

What lessons can we learn from the Ragusa study, and what is the impact on treatment of CRC in humans? The study provides a clear demonstration of what we already know: there is no magic bullet in cancer treatment. Drugs have to be combined in a mix, tailored to fit each individual's cancer. The study also points to the critical balance between the tumor and nontumor cell compartments and how cells in transit may visit and steer the development of the disease in very different directions (8). Thereby, under certain treatment conditions, the immune system can launch antitumor reactions against cancer types resistant to checkpoint inhibition due to lack of cytotoxic T cells in the tumor. The study, moreover, renews the interest in targeting MMP14 in cancer, which, through novel strategies such as computational design, may eventually be successful (18). Finally, the study provides hope for finding new therapy designs for hard-to-treat, chemotherapy-resistant cancer for which little can be offered today.

\section{Acknowledgments}

The author acknowledges support from the Swedish Cancer foundation (19 0119 Pj $01 \mathrm{H})$.

Address correspondence to: Lena Claesson-Welsh, Rudbeck Laboratory, Department of Immunology, Genetics and Pathology, Dag Hammarskjölds väg 20, 75185 Uppsala, Sweden. Phone: 46.70.167.9260; Email: lena.welsh@igp.uu.se.

1. Gross J, Lapierre CM. Collagenolytic activity in amphibian tissues: a tissue culture assay. Proc Natl Acad Sci U S A. 1962;48:1014-1022.

2. Rodríguez D, Morrison CJ, Overall CM. Matrix metalloproteinases: what do they not do? New substrates and biological roles identified by murine models and proteomics. Biochim Biophys Acta. 2010;1803(1):39-54.

3. Chelladurai P, Seeger W, Pullamsetti SS. Matrix metalloproteinases and their inhibitors in pulmonary hypertension. Eur Respir J. 2012;40(3):766-782.

4. Gutiérrez-Fernández A, et al. Loss of MT1-MMP causes cell senescence and nuclear defects which can be reversed by retinoic acid. EMBO J. 2015;34(14):1875-1888.

5. Sato $\mathrm{H}$, et al. A matrix metalloproteinase expressed on the surface of invasive tumour cells. Nature. 1994;370(6484):61-65.

6. Wieczorek E, Jablonska E, Wasowicz W, Reszka E. Matrix metalloproteinases and genetic mouse models in cancer research: a mini-review. Tumour Biol. 2015;36(1):163-175.

7. Winer A, Adams S, Mignatti P. Matrix metalloproteinase inhibitors in cancer therapy: turning past failures into future successes. Mol Cancer Ther. 2018;17(6):1147-1155.

8. Ragusa $S$, et al. Antiangiogenic immunotherapy 
suppresses desmoplastic and chemoresistant intestinal tumors in mice. JClin Invest. 2020;130(3):1199-1216.

9. Gramolelli S, et al. PROX1 is a transcriptional regulator of MMP14. Sci Rep. 2018;8(1):9531.

10. Queirolo P, Boutros A, Tanda E, Spagnolo F, Quaglino P. Immune-checkpoint inhibitors for the treatment of metastatic melanoma: a model of cancer immunotherapy. Semin Cancer Biol. 2019;59:290-297.

11. Ghiringhelli F, Fumet JD. Is there a place for immunotherapy for metastatic microsatellite stable colorectal cancer? Front Immunol. 2019;10:1816.
12. Martin JD, Seano G, Jain RK. Normalizing function of tumor vessels: progress, Opportunities, and Challenges. Annu Rev Physiol. 2019;81:505-534.

13. Schmittnaegel M, et al. Dual angiopoietin-2 and VEGFA inhibition elicits antitumor immunity that is enhanced by PD-1 checkpoint blockade. Sci Transl Med. 2017;9(385):eaak9670.

14. Rosen LS, Jacobs IA, Burkes RL. Bevacizumab in colorectal cancer: current role in treatment and the potential of biosimilars. Target Oncol. 2017;12(5):599-610.

15. Fukumura D, Kloepper J, Amoozgar Z, Duda DG, Jain RK. Enhancing cancer immunotherapy using antiangiogenics: opportunities and chal- lenges. Nat Rev Clin Oncol. 2018;15(5):325-340.

16. Vonderheide RH. CD40 agonist antibodies in cancer immunotherapy [published online ahead of print August 14, 2019]. Anпи Rev Med. https:// doi.org/10.1146/annurev-med-062518-045435.

17. Allen $\mathrm{E}$, et al. Combined antiangiogenic and anti-PD-L1 therapy stimulates tumor immunity through HEV formation. Sci Transl Med. 2017;9(385):eaak9679.

18. Arkadash V, et al. Development of high affinity and high specificity inhibitors of matrix metalloproteinase 14 through computational design and directed evolution. J Biol Chem. 2017;292(8):3481-3495. 